\title{
Finite-element Modeling of Elastic Surface Modes and Scattering from Spherical Objects
}

\author{
Omar Falou ${ }^{*}, 1$ J. Carl Kumaradas ${ }^{2}$, and Michael C. Kolios ${ }^{1,2}$ \\ ${ }^{1}$ Dept. of Electrical and Computer Engineering, Ryerson University, ${ }^{2}$ Dept. of Physics, Ryerson \\ University \\ *Corresponding author: 350 Victoria Street, Toronto, Ontario M5B 2K3, Canada, ofalou@ryerson.ca
}

\begin{abstract}
A finite-element model of wave propagation using COMSOL Multiphysics (COMSOL Inc., Burlington, MA) was developed to solve the problem of high frequency ultrasound scattering from spheres. This model is used to predict ultrasound backscatter from cells for ultrasound tissue characterization. In this work, the backscatter from an elastic sphere was used to validate the computational model against analytical solutions (Faran theory). Agreements between analytical and finite element solutions were found in the scattered far-field over a range of frequencies of interest $(10-70 \mathrm{MHz})$. Oscillations of the elastic sphere at various resonance frequencies (peaks in the power spectrum) were also investigated. The resonance frequencies predicted by the analytical solutions corresponded to surface modes. A systematic relationship between the resonance frequency and its corresponding surface mode was found. The oscillations of the elastic sphere were visualized at these resonances. An ultrasound scattering model by a single cell is also presented. The model treats the cell as an elastic sphere (nucleus) surrounded by a fluid shell (cytoplasm). Comparison of the theoretical backscatter predicted by the model and experimental measurements for Acute Myeloid Leukemia (AML) cell is also shown. Finally, the implications of these results on the prediction of ultrasound backscatter from cells, and on ultrasound tissue characterization techniques are discussed.
\end{abstract}

Keywords: Ultrasound imaging, acoustic scattering, elastic surface modes, cell scattering

\section{Introduction}

It has been shown that high frequency ultrasound $(20 \mathrm{MHz}-60 \mathrm{MHz})$ can be used to detect structural and physical changes in cell ensembles during cell death (Sherar et al. 1987), including apoptosis (Czarnota et al. 1997;
Czarnota et al. 1999; Kolios et al. 2004). Ultrasonic backscatter from cell ensembles treated with the chemotherapeutic cisplatin, which induces apoptosis, increased the ultrasound backscatter by $9-13 \mathrm{~dB}$ resulting in much brighter images. However, the precise physical mechanism that causes this increase is not known.

A theoretical model of acoustic wave scattering is required to better understand scattering from cells, which would then allow us to determine the proportion of cells undergoing apoptosis in a given ensemble. The effect of the morphological changes during apoptosis on ultrasound backscatter cannot be modeled using analytical methods. Most scattering models used for lower frequency ultrasonic tissue characterization assume a random distribution of scatterers (Lizzi et al. 1983; Insana et al. 1990; Oelze et al. 2002). These models have been used to diagnose various tissue pathologic states (Lizzi et al. 1988; Lizzi et al. 1997; Ursea et al. 1998; Feleppa et al. 2000). However, they do not take into account the elastic nature of cells, which may be significant at higher frequencies. There is evidence of an elastic nature to cells with a variation in elasticity within the cell (Caille et al. 2002; Zinin et al. 2005). For example, the cell nucleus has been shown to be stiffer (Tseng et al. 2004) and more viscous (Guilak et al. 2000) than the cytoplasm.

Moreover, there is an increasing interest in the modeling of ultrasound contrast agents' oscillations, collapse, and scattering in ultrasound fields for a better understanding of contrast agent imaging (Stride et al. 2003). Current models used cannot easily take into account features such as non-radial bubble oscillations due to inhomogeneity and the interactions of bubbles with their surroundings.

Analytical solutions to the problem of wave scattering from simple, isotropic, and homogeneous three dimensional structures such as spheres have been studied extensively in the 
past (Anderson 1950; Faran 1951; Hickling 1962). However, these formulations are not flexible enough to be extended to wave scattering from complex scatterers such as cells which exhibit irregular shape and heterogeneous composition. Baddour et al. (2005) made the first attempt to build such a model for OCIAML-5 (Acute Myeloid Leukemia) (Wang et al. 1991) cells based on Faran's formulation (Faran 1951) by assuming that scattering occurs from the nucleus of a cell. They assumed that the nucleus was homogenous and spherical. The analytical model failed to accurately predict the experimentally measured backscatter from cells. Therefore, a more complete cell model which incorporates the mechanical properties of various cell components is needed.

In our previous work (Falou et al. 2005), a finite-element model of acoustic wave propagation was developed to solve the problem of acoustic scattering from spheres. The model was shown to accurately predict near-field scattering.

In this work, a comparison of finite-element and analytical solutions in the far-field is given. Moreover, a study of surface modes exhibited by an elastic microsphere subject to various frequencies is presented. Finally, a scattering model of a single cell which treats the cell as an elastic nucleus surrounded by a fluid cytoplasm is shown and compared with experimental measurements.

\section{Methods}

\subsection{Far-field Backscatter Calculation}

Given the pressure and its normal derivative on a spherical surface surrounding the scatterer, the Helmholtz Integral equation can be used to obtain the far-field backscattered pressure. The Helmholtz Integral equation (Junger et al. 1993) is given by:

$$
p(\boldsymbol{R})=-\int_{S_{0}}\left(p \frac{\partial g}{\xi_{0}}+\rho \ddot{w} g\right) d S\left(\boldsymbol{R}_{\boldsymbol{o}}\right)
$$

where, $\boldsymbol{R}$ and $\boldsymbol{R}_{\boldsymbol{0}}$ are the position vectors locating field and source points, respectively. $\xi_{0}$ is the outward pointing unit vector normal to the radiating spherical surface, $\boldsymbol{\rho}$ is the density of the medium, and $\ddot{\boldsymbol{w}}$ is the normal component of acceleration at the surface $\boldsymbol{S}_{\boldsymbol{0}} . \boldsymbol{g}$ is the free-space Green's function defined as:

$$
g\left(\left|\boldsymbol{R}-\boldsymbol{R}_{\boldsymbol{0}}\right|\right)=-\frac{e^{i k\left|\boldsymbol{R}-\boldsymbol{R}_{\theta}\right|}}{4 \pi\left|\boldsymbol{R}-\boldsymbol{R}_{\boldsymbol{0}}\right|}
$$

The far-field backscatter transfer functions BSTF (Baddour et al. 2005) calculated using the finite-element model were compared with analytical solutions (Faran 1951) for a 11.5 micron polystyrene microsphere immersed in phosphate buffered saline (PBS) having a density of $1000 \mathrm{Kg} / \mathrm{m}^{3}$ and speed of sound of $1483 \mathrm{~m} / \mathrm{s}$ (Baddour et al. 2005). Table 1 gives the physical properties of the polystyrene microsphere.

Table 1: Physical properties of polystyrene

\begin{tabular}{|l|c|}
\hline Density & $1050 \mathrm{Kg} / \mathrm{m}^{3}$ \\
\hline Speed of sound & $2350 \mathrm{~m} / \mathrm{s}$ \\
\hline Poisson's Ratio & 0.35 \\
\hline
\end{tabular}

\subsection{Surface Modes of a Polystyrene Microsphere}

The BSTF versus frequency for a 43 micron polystyrene microsphere was plotted to determine the microsphere resonance frequencies corresponding to peaks in the plot. Oscillations representing surface modes of the microsphere at various resonance frequencies were then visualized by plotting particle's displacement at the sphere's surface using COMSOL Multiphysics 3.3a (COMSOL Inc., Burlington, MA).

\begin{tabular}{|c|c|c|}
\hline \multirow{4}{*}{$\frac{\tilde{0}}{\frac{0}{0}}$} & Diameter & $9.1 \mu \mathrm{m} \S$ \\
\hline & Density & $1430 \mathrm{Kg} / \mathrm{m}^{3} \S$ \\
\hline & Speed of sound & $1503 \mathrm{~m} / \mathrm{s} \ddagger$ \\
\hline & Poisson's Ratio & $0.42 *$ \\
\hline \multirow{3}{*}{ 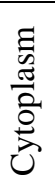 } & Diameter & $11.5 \mu \mathrm{m} \S$ \\
\hline & Density & $1050 \mathrm{Kg} / \mathrm{m}^{3+}$ \\
\hline & Speed of sound & $1535 \mathrm{~m} / \mathrm{s}+$ \\
\hline
\end{tabular}

Table 2: Physical properties of AML cell model

$\$$ (Baddour et al. 2005)

(Taggart et al. 2006). Speed of sound in cytoplasm is assumed to be same as that in the whole cell

* (Knight et al. 2002).Assumed to be the same as that of nuclei of chondrocytes

${ }^{+}$Cytoplasm assumed to be water-like low concentration saline 


\subsection{Proposed Cell Model}

This section presents an ultrasound scattering model by a single AML cell. The model treats the cell as an elastic sphere (nucleus) surrounded by a fluid shell (cytoplasm). Table 2 lists the physical properties of the cell used in the simulations.

Comparison of the theoretical backscatter predicted by the model and experimental measurements (Baddour et al. 2007) for Acute Myeloid Leukemia (AML) cell is also shown.

\section{Results and Discussion}

Figure 1 compares the finite-element and analytical solutions of the far-field backscatter transfer function versus frequency for an 11.5 micron polystyrene microsphere immersed in PBS. There is a good agreement between the analytical and finite element solution, despite the sharp changes in pressure as a function of frequency.

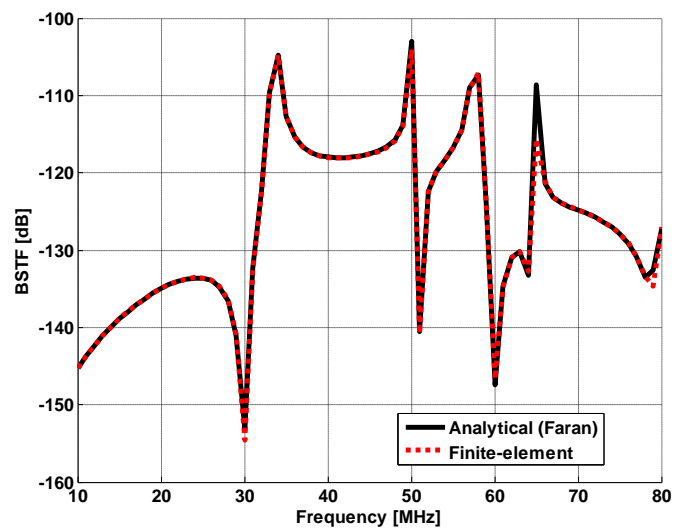

Figure 1. Analytical (Faran) and finite-element solutions for scattering from an 11.5 micron polystyrene microsphere immersed in PBS. The plot shows the far-field backscatter transfer functions [dB] versus frequency $[\mathrm{MHz}]$.

Figure 2 shows the backscatter transfer function versus frequency for a 43 micron polystyrene microsphere immersed in PBS. Various peaks in the backscatter are observed which correspond to the sphere's resonant frequencies. The first 5 of the latter were found to be at 16.1, 23.5, 30.5, 37.3, and $44 \mathrm{MHz}$.

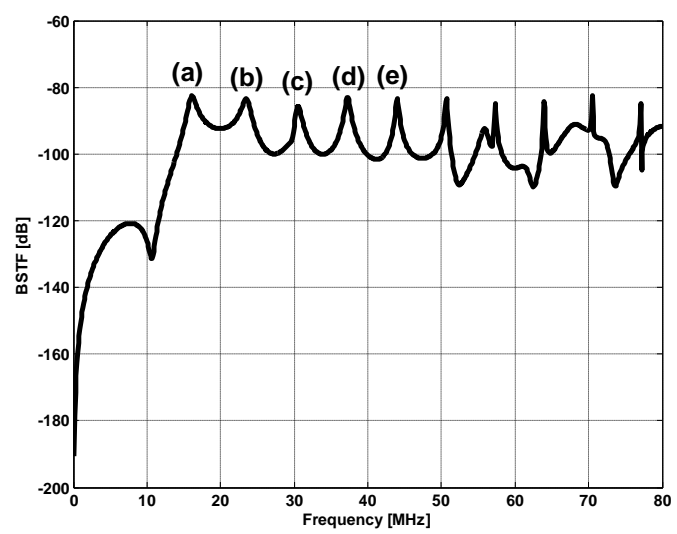

Figure 2. Analytical (Faran) and finite-element solutions for scattering from a 43 micron polystyrene microsphere immersed in PBS. The plot shows the farfield backscatter transfer functions $[\mathrm{dB}]$ versus frequency $[\mathrm{MHz}]$.

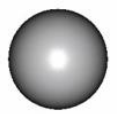

(a)

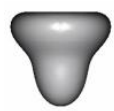

(b)

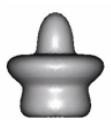

(d)

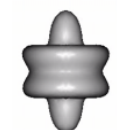

(e)
Figure 3. Finite-element solutions for deformations of a 43 micron polystyrene microsphere at a) 16.1, b) 23.5 , c) 30.5 , d) 37.3 , and e) $44 \mathrm{MHz}$. The plots show a snapshot of the deformation for a slice parallel to the direction of wave propagation (bottom to top). The displacement was multiplied by a factor of $10^{8}$ to $10^{9}$ for visualization purposes.

Figure 3 depicts the deformations of the polystyrene microsphere immersed in PBS at 5 resonance frequencies which corresponded to surface modes often represented by an integer $n$, where $n=0$ corresponds to the radial oscillation, $n=2$ to an ellipsoid, etc. It can be seen that there is a systematic relationship between the resonance frequency and its corresponding surface mode. The higher the resonance frequency, the more complex the oscillation pattern, and hence, the higher the mode number. Knowledge of such deformations is important since it may help in understanding the process of bubble rupture in the study of ultrasound contrast agents.

Figure 4 compares the theoretical BSTF versus frequency predicted by the finite-element model with experimental measurements for 
AML cells. It is evident that the proposed model does not accurately predict the backscatter from AML cells. This may due to several reasons: firstly, assumptions were made regarding the physical properties of AML cells as precise measurements are not available in the literature. For instance, Poisson's ratio (the ratio of strain in the lateral direction to strain in the axial direction) of AML cells assumed to be the same as that of another cell type, chondrocytes, which may not be the case. Our work has shown that a small variation in the Poisson's ratio may substantially affect the backscattered field. Secondly, this model doesn't take into account the viscosity of the AML cell and nucleus which may affect the backscatter through the process of damping. Lastly, the proposed model may be oversimplified since does not take into account the presence of the cell membranes and large organelles in the cytoplasm such as, mitochondria, which may also affect the backscatter.

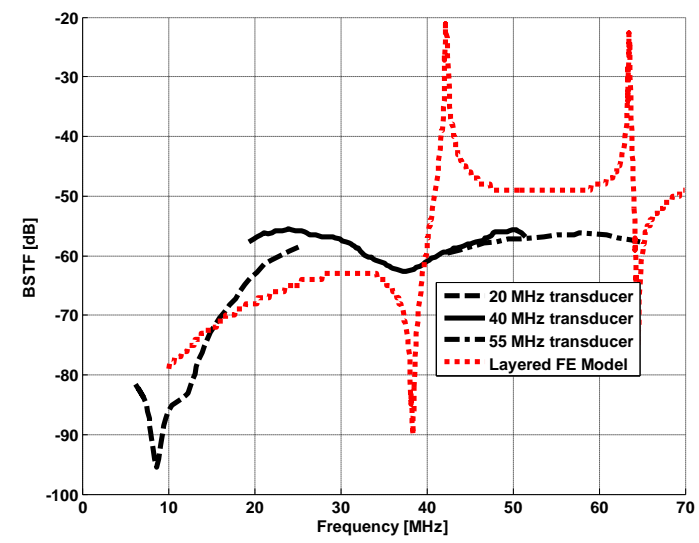

Figure 4. Finite element cell model versus experimental measurements. The plot shows the backscatter transfer functions $[\mathrm{dB}]$ versus frequency $[\mathrm{MHz}]$. The finite element plot was shifted by $+70 \mathrm{~dB}$ for visualization purposes.

\section{Conclusions and Future work}

Agreement between analytical and finiteelement solutions was found in the scattered farfield over a range of frequencies of interest (10 $70 \mathrm{MHz}$ ). Oscillations of an elastic sphere at various resonance frequencies were investigated. A systematic relationship between the resonance frequency and its corresponding surface mode was found. An ultrasound scattering model by a single cell was also presented. The model treats the cell as an elastic sphere (nucleus) surrounded by a fluid shell (cytoplasm). Comparison of the theoretical backscatter predicted by the model and experimental measurements for Acute Myeloid Leukemia (AML) cell did not show a good agreement. Reasons for such discrepancies were discussed.

Future work includes the modeling of backscatter and oscillations from ultrasound contrast agents and the refinement of the finiteelement cell model.

\section{References}

1. Anderson, V. C., Sound scattering from a fluid sphere, Journal of the Acoustical Society of America 22(4): 426-431, (1950)

2. Baddour, R. E. and M. C. Kolios, The fluid and elastic nature of nucleated cells: Implications from the cellular backscatter response, Journal of the Acoustical Society of America 121(1): EL16EL22, (2007)

3. Baddour, R. E., M. D. Sherar, et al., Highfrequency ultrasound scattering from microspheres and single cells, Journal of the Acoustical Society of America 117(2): 934-943, (2005)

4. Caille, N., O. Thoumine, et al., Contribution of the nucleus to the mechanical properties of endothelial cells, Journal of Biomechanics 35(2): 177-187, (2002)

5. Czarnota, G. J., M. C. Kolios, et al., Ultrasound imaging of apoptosis: Highresolution non-invasive monitoring of programmed cell death in vitro, in situ and in vivo, British Journal of Cancer 81(3): 520-527, (1999)

6. Czarnota, G. J., M. C. Kolios, et al., Ultrasonic biomicroscopy of viable, dead and apoptotic cells, Ultrasound in Medicine and Biology 23(6): 961-965, (1997)

7. Falou, O., J. C. Kumaradas, et al. A study of femlab for modeling high frequency ultrasound scattering by spherical objects. FEMLAB User Conference, Boston, MA, (2005)

8. Faran, J. J., Sound scattering by solid cylinders and spheres, Journal of the Acoustical Society of America 23(4): 405-418, (1951)

9. Feleppa, E. J., T. Liu, et al. Three-dimensional ultrasonic parametric and tissue-property imaging for tissue evaluation, treatment planning, therapy guidance, and efficacy assessment. Proceedings of SPIE - The 
International Society for Optical Engineering, San Diego, CA, USA, (2000)

10. Guilak, F., J. R. Tedrow, et al., Viscoelastic properties of the cell nucleus, Biochemical and Biophysical Research Communications 269(3): 781-786, (2000)

11. Hickling, R., Analysis of echoes from a solid elastic sphere in water, Journal of the Acoustical Society of America 34(10): 1582-1592, (1962)

12. Insana, M. F. and T. J. Hall, Characterizing the microstructure of random-media using ultrasound, Physics in Medicine and Biology 35(10): 1373-1386, (1990)

13. Junger, M. C. and D. Feit, Sound, structures, and their interaction, Acoustical Society of America.

14. Kaighn, M. E., K. S. Narayan, et al., Establishment and characterization of a human prostatic-carcinoma cell-line ( $\mathrm{pc}-3)$, Investigative Urology 17(1): 16-23, (1979)

15. Knight, M. M., J. Bravenboer, et al., Cell and nucleus deformation in compressed chondrocytealginate constructs: Temporal changes and calculation of cell modulus, Biochimica Et Biophysica Acta-General Subjects 1570(1): 1-8, (2002)

16. Kolios, M. C., G. J. Czarnota, et al. Towards understanding the nature of high frequency backscatter from cells and tissues: An investigation of backscatter power spectra from different concentrations of cells of different sizes. 2004 IEEE International Ultrasonics, Ferroelectrics, and Frequency Control Joint 50th Anniversary Conference, (2004)

17. Lizzi, F. L., M. Astor, et al., Statistical framework for ultrasonic spectral parameter imaging, Ultrasound in Medicine and Biology 23(9): 1371-1382, (1997)

18. Lizzi, F. L., M. Greenebaum, et al., Theoretical framework for spectrum analysis in ultrasonic tissue characterization, Journal of the Acoustical Society of America 73(4): 1366-1373, (1983)

19. Lizzi, F. L., D. L. King, et al., Comparison of theoretical scattering results and ultrasonic data from clinical liver examinations, Ultrasound in Medicine and Biology 14(5): 377-385, (1988)

20. Oelze, M. L., J. F. Zachary, et al., Characterization of tissue microstructure using ultrasonic backscatter: Theory and technique for optimization using a gaussian form factor, Journal of the Acoustical Society of America 112(3): 1202-1211, (2002)
21. Sherar, M. D., M. B. Noss, et al., Ultrasound backscatter microscopy images the internal structure of living tumor spheroids, Nature 330(6147): 493-495, (1987)

22. Stride, E. and N. Saffari, Microbubble ultrasound contrast agents: A review, Proceedings Of The Institution Of Mechanical Engineers Part H-Journal Of Engineering In Medicine 217(H6): 429-447, (2003)

23. Taggart, L., R. E. Baddour, et al., Ultrasonic characterization of viable whole cells and isolated nuclei, Ultrasound in Medicine and Biology: in press, (2006)

24. Tseng, Y., J. S. H. Lee, et al., Microorganization and visco-elasticity of the interphase nucleus revealed by particle nanotracking, Journal of Cell Science 117(10): 2159-2167, (2004)

25. Ursea, R., D. J. Coleman, et al., Correlation of high frequency ultrasound backscatter with tumor microstructure in iris melanoma, Ophthalmology 105(5): 906-912, (1998)

26. Wang, C., P. Koistinen, et al., Mast-cell growth-factor, a ligand for the receptor encoded by c-kit, affects the growth in culture of the blast cells of acute myeloblastic-leukemia, Leukemia 5(6): 493-499, (1991)

27. Zinin, P. V., J. S. Allen, et al., Mechanical resonances of bacteria cells, Physical Review $E$ 72(6), (2005)

\section{Acknowledgments}

The authors acknowledge Ralph Baddour of the Department of Medical Biophysics at the University of Toronto for helpful technical discussions. This work was supported by the Canadian Institutes of Health Research (CIHR), and Natural Sciences and Engineering Research Council of Canada (NSERC). The high frequency ultrasound instrument used in the work was purchased with funds from the Canada Foundation for Innovation (CFI), the Ontario Ministry of Research and Innovation, and Ryerson University. 